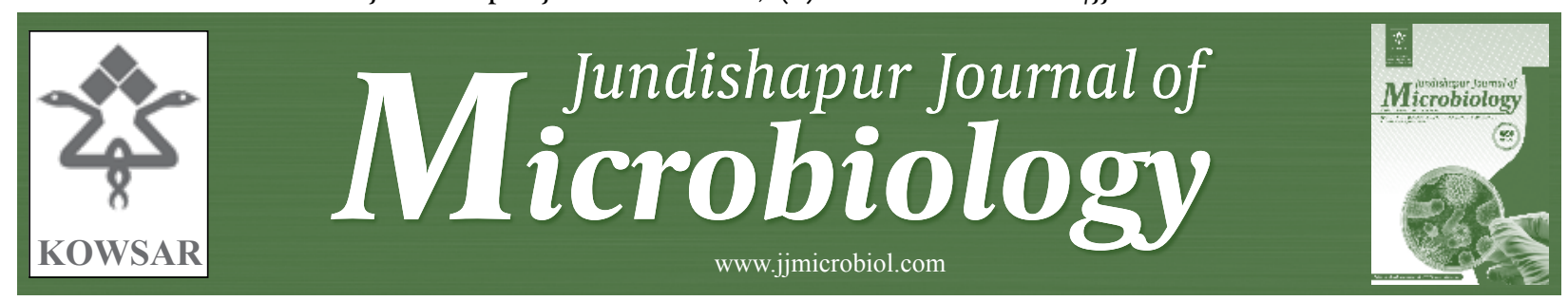

\title{
Prevalence of HBsAg, HCV and HIV Antibodies Among Infertile Couples in Ahvaz, South-West Iran
}

\author{
Roshan Nikbakht ${ }^{1}$, Nasrin Saadati ${ }^{*}$, Farzam Firoozian ${ }^{3}$ \\ ${ }^{1}$ Department of Obstetrics and Gynecology, Imam Khomeini Hospital, Fertility and Infertility Research Center, Ahvaz Jundishapur University of Medical \\ Sciences, School of Medicine, Ahvaz, IR Iran \\ 2 Department of Community Medicine, School of Medicine, Fertility and Infertility Research Center, Ahvaz Jundishapur University of Medical Sciences, \\ Ahvaz, IR Iran \\ 3 General Practitioners of Ahvaz Jundishapur University of Medical Sciences, Ahvaz, IR Iran
}

A R T I C L E I N F O

Article type:

Original Article

Article history:

Received: 03 Dec 2010

Revised: 20 Jan 2011

Accepted: 01 Jun 2011

Keywords:

Hepatitis B Surface Antigen (HBsAg)

Anti-HCV Antibodies

Anti-HIV Antibodies

Infertile Couples \begin{abstract}
A B S T R A C T
Background: Without doubt the problem of infertility is important not just for the individual couple, but in many cases it has a wider effect on human life which may lead to social disorganization if not addressed in the future if not addressed in the future.

Objectives: Since the screening of the hepatitis B surface antigen (HBsAg), hepatitis C virus (HCV) and human immunodeficiency virus (HIV) antibodies of infertile couples has not been studied in our area; this study was conducted to determine the prevalence of HBsAg, HCV \& HIV antibodies among infertile couples.

Patients and Methods: This was a retrospective cross sectional study and its setting was an in vitro fertilization (IVF) ward. The population study included all of the couples (712) admitted to the infertility center of Ahvaz Imam Khomeini Hospital in 2007-2008 (12 month period). Intervention was an analysis of the data containing the patients' demographic characteristics and included their HBsAg, HCV and HIV serostatus, which were routinely screened during this period. The main outcome measure was the prevalence of HBsAg, HCV and HIV antibodies among the infertile couples referred to the infertility center.

Results: The age range of the men and women was 18 to 62 years and 16 to 46 years respectively. $11(0.77 \%)$ of the infertile couples, including $6(0.8)$ women and $5(0.7 \%)$ men were HBsAg positive. $9(0.63 \%)$ infertile couples, $6(0.8)$ women and $3(0.4 \%)$ men were $\mathrm{HCV}$ antibody positive. No cases of HIV were observed in this study.

Conclusions: Although the frequencies of hepatitis B and C infections found in this study were small, it still seems logical from the statistical analysis to screen for both viruses as well as for HIV, while a future study using a larger sample size of infertile couples is also recommended.
\end{abstract}

Copyright $\odot 2012$ Kowsar Corp. All rights reserved.

\section{- Implication for health policy/practice/research/medical education:}

The present study determined some critical information about the prevalence of HBsAg, HCV \& HIV antibodies among infertile couples in southern Iran.

Please cite this paper as:

Nikbakht R, Saadati N, Firoozian F. Prevalence of HBsAg, HCV and HIV Antibodies Among Infertile Couples in Ahvaz, South-West Iran. Jundishapur J Microbiol. 2012;5(2):393-7. DOI: 10.5812/jjm.2809

* Corresponding author: Nasrin Saadati, Department of Community Medicine, School of Medicine, Ahvaz Jundishapur University of Medical Sciences, Ahvaz, IR Iran. Tel: +98-6113361518, Fax: +98-6113332036, E-mail: NSaadaty@ajums.ac.ir

DOI:10.5812/jjm.2809

Copyright $\odot 2012$ Kowsar Corp. All rights reserved.

\section{Background}

Infertility is defined as the failure of a couple to conceive after 12 months of regular intercourse without the use of contraception. Demand for infertility services has grown substantially even though the prevalence of in- 
fertility has remained stable (1-3). However the problem of infertility is important to society as well as for the affected individuals and the $10-15 \%$ prevalence of infertility also underlines the importance of this issue (4). The American Society for Reproductive Medicine has published guidelines on reproductive issues in women and men with hepatitis $(5,6)$. Two billion people worldwide show evidence of hepatitis B virus exposure, and it is estimated that 400 million people are actively infected and affected by this infection (7). Approximately 1.5 million people in Iran are living with hepatitis B virus (HBV) infection (mild to moderate prevalence according to WHOclassification), the HBV infection prevalence in Iran is estimated to be $2.14 \%$ (95\% CI:1.92-2.35).

The HBV infection rate in Iranian men and women is estimated to be $2.55 \%$ (95\% CI: $2.25-2.85$ ) and 2.03\% (95\% CI: 1.6-2.46\%), respectively (8). Screening for HBV before assisted reproductive treatment (ART) is an increasingly common practice in most centers $(9,10)$. Pirwany et al. (11) examined the reproductive performance of 13 couples discordant for HBV (10 men and 3 women) and 27 controls, after their first IVF and embryo transfer treatment. The small retrospective study showed that couples, one of whom is infected with HBV, had much lower pregnancy rates (PR) $(7.7 \%$ vs. $40.7 \%$; $P<0.01)$ than their age-matched controls (11). All neonates born to hepatitis B surface antigen (HBsAg) positive women should receive hepatitis $B$ immunoglobulin (HBIg) and hepatitis B vaccine at birth. More than 200 million people worldwide are infected with the hepatitis $\mathrm{C}$ virus (HCV) ; following the primary infection, $60-88 \%$ of these cases become chronic (12). It would appear that the prevalence of HCV infection in the Iranian population is $0.16 \%$ (95\% CI: 0\%-0.59\%) (13).

The seroprevalence of HCV antibodies in pregnancy has been determined to be from $0.4 \%$ to $1.86 \%(14,15)$. With the improvements in life expectancy and quality of life for HIV-infected individuals in the developed world, many wish to have children, so reproductive issues are becoming increasingly important. Reproductive technologies provide a logical way to minimize HIV transmission for HIV couples desiring pregnancy. Although the most recent research is compelling, much more is needed and protocols developed in order to establish the safety of these techniques which will assist physicians to better serve these patients (16). In couples affected by HIV, an acceptable pregnancy rate was obtained, but the lowest results were obtained when both partners were infected (17).

HIV infection is now considered to be a chronic disease, so all couples who are affected by HIV, whether fertile or infertile, who want to have genetically related offspring should be seen before conception for counseling and testing. There is no published data assessing the welfare of the child in HIV positive couples undergoing assisted conception. The authors present a rather comprehensive review of the literature for HBsAg, HCV and HIV affected couples. They provide existing evidence that the problem is likely to be present and sought to determine the prevalence of HBsAg, HCV and HIV antibodies among infertile couples. HIV and hepatitis B and C are viral diseases which require special attention to their risk factors and the systemic nature of the disease, while complications such as chronic or high mortality from them are also very important. According to research it seems on the surface that community awareness of ways to prevent AIDS and hepatitis transmission is low and so health workers need to pay more importance to this issue (18).

\section{Objectives}

The objective of this study was to determine the prevalence of HBsAg, HCV and HIV infection among infertile couples. All (712) of the couples admitted during this period, were screened for HBsAg, HCV and HIV antibodies before evaluation. There is no baseline data on the distribution of HBV, HCV and HIV among infertile couple in Ahvaz, so in this retrospective study we sought to determine the prevalence of HBV, HCV and HIV infection.

\section{Patients and Methods}

We performed a retrospective cross sectional study to examine the prevalence of HBV, HCV and HIV infection. The study subjects included all (712) infertile couples referred for IVF at the Ahvaz Imam Khomeini Hospital from March 2007 until March 2008.This hospital is the main referral center for infertile couples in Ahvaz City, Iran. All subjects involved gave their informed consent to participate and to have their blood samples tested. The data was collected through completing a questionnaire; as a supplementary tool to accompany the laboratory test results.

Sera from patients were screened for HBsAg determined with an ELISA (enzyme-linked immunosorbent assay )and blood virus screening anti-HCV with an ELISA German Kit (Golden Technologies, LLC / Germany Kit Two - step Incubation, indirect Principle Instruction- Dmitry Trofimov Institute of Immunology Russia-dima@dna- technology.ru) was used (19). Antibodies to HIV were tested by an ELISA German Kit for qualitative detection of (HIV1/2). Statistical analyses were performed using the Statistical Package for the Social Sciences, version 16.0 (SPSS Inc. Chicago, IL).

\section{Results}

The age range of the men and women was 18 to 62 years and 16 to 46 years respectively. The mean age for females was $27.98 \pm 5.34$ and males $33.13 \pm 6.05$ years. $35.5 \%$ of the females and $35.4 \%$ of the males were $26-30$ years old (Table 1). $81.1 \%$ of the infertile couples had a primary type of infertility (Table 2). 11 (0.77\%) infertile couples; 6 (0.8) women and $5(0.7 \%)$ men were $\mathrm{HBV}$ positive, while 9 (0.63 $\%$ ) infertile couples; 6 (0.8) women and $3(0.4 \%)$ men were 


\begin{tabular}{lll}
\hline \multicolumn{3}{l}{ Table 1. Infertile Couples (Participants) According to Age and Sex } \\
\hline Age, $\mathbf{y}$ & Female $(\mathbf{n}=\mathbf{7 1 2})$ & Male $(\mathbf{n}=\mathbf{7 1 2})$ \\
\hline$\leq 20$ & $28(3.8)$ & $1(0.1)$ \\
$21-25$ & $250(34)$ & $31(4.2)$ \\
$26-30$ & $261(35.5)$ & $260(35.4)$ \\
$31-35$ & $123(16.7)$ & $221(30.1)$ \\
$36-40$ & $48(5.6)$ & $149(20.3)$ \\
$\geq 41$ & $25(3.4)$ & $73(9.9)$ \\
Mean \pm SD & $27.98 \pm 5.34$ & $33.13 \pm 6.05$ \\
\hline
\end{tabular}

\begin{tabular}{lll}
\hline \multicolumn{2}{l}{ Table 2. Infertile Couples (Participants) According to Type of Infertility } \\
\hline & Frequency & No.\% \\
\hline Primary & 570 & 81.1 \\
Secondary & 133 & 18.9 \\
Total & 703 & 100 \\
\hline
\end{tabular}

HCV positive. No cases of HIV population were observed in this study.

\section{Discussion}

In this study the prevalence of HBsAg, HCV and HIV among infertile couples referred to the IVF Department of Ahvaz Imam Khomeini Hospital, during the year was, respectively, $0.77 \%$ and $0.63 \%$ and $0.0 \%$. Attention needs to be given to the impact of HIV on clinical infertility practice, it is important to have a cautious and flexible approach to minimize the risk to infertile couples and to future children. In a study by Hargreaves over five years in an Edinburgh infertility center, no cases of HIV infection were observed among any of the referrals (20).

Prisant et al. evaluated the viral infection effects on infertility treatment outcomes in HIV-1 or HCV monoinfected infertile serodiscordant couples, in a retrospective case-controlled study. Between December 2002 and June 2007, 232 cycles of IVF (In Vitro Fertilization) /ICSI (Intracytoplasmic sperm injection) for 130 serodiscordant couples (SDC) were compared with 232 cycles for 211 matched seronegative couples. Clinical pregnancy rates for HIV-1 or HCV infertile serodiscordant couples were not significantly different from those of the seronegative controls (21). Increased longevity and quality of life has led some HIV-positive patients to think about childbearing, but careful preconception counseling is necessary (22). Townsend and his group analyzed the Comprehensive national surveillance study to explore the impact of different strategies to prevent mother-to-child HIV transmission in the United Kingdom and Ireland between 2000 and 2006.The overall mother-to-child transmission rate was $1.2 \%$ (61/5151, 95\% CI: 0.9-1.5\%), and 0.8\% (40/4864) for the women who received at least 14 days of antiretroviral therapy (23).

Screening for HBV before assisted reproductive treat- ment (ART) is necessary, as transplacental transmission due to leakage can occur, for example during a threatened abortion. Transmission at birth is more likely if the mother is hepatitis B or HBsAg positive. Knowledge of the couple's serostatus allows immunoprophylactic measures to be taken; first to reduce the risk of virus transmission to the partner or fetus, and second precautions need to be taken against cross-contamination during sample handing and embryo cryo storage (Cryo storage of embryo). It also enables couples to make an informed decision regarding whether to pursue treatment. Vertical transmission may occur from a HBV seropositive mother as a consequence of intrauterine exposure, transplacental transmission, or breastfeeding. Interestingly, HBV infection has also been detected in the newborns of HBV seronegative mothers, because HBV DNA has been detected in semen and spermatozoa (24), and it is inferred that father neonate transmission of HBV might be possible (25). More studies need to be concentrated on detection of the HBV genome in gametes and embryos. With greater knowledge of the vertical transmission processes of HBV, better preconception counseling can be offered to HBV seropositive couples (9). Moreover, some mutant HBV remains undetectable by serological tests.

In a prospective cross-sectional descriptive study, 250 hemodialysis patients from the Khuzestan Province were selected at least six (months, from winter 2008 to spring 2009.Patients suffering from HCV, hepatitis D virus (HDV) or HIV co-infection were excluded from the study. The prevalence of HBV infection was high among the dialysis patients (20\%), and occult hepatitis B was $4 \%$. The dominant genotype of HBV was D2 (92\%) followed by genotypes B6 (4\%) (26). Another descriptive study was carried out to estimate the frequency of HBsAg by a simple sampling method of 120 pregnant women referred to health centers in Ahvaz City from March to December 1998.In order to determine hepatitis B surface antigen, an in vitro diagnostic kit called Hepanostika-HBsAg, which is an enzyme immunoassay, was used. The research showed that 2 out of 120 (1.7\%) specimens were positive for HBsAg with no evidence of HBeAg $(27,28)$. Mboto et al. (14) in their study found that the prevalence of HCV was $0.4 \%$ among a total of 506 out of 716 antenatal care, pregnant women in Calabar City. This study revealed a low rate of HCV.

Onakewhor and Okonofua (15) in a prospective crosssectional study of 269 subjects who were consecutively booked, antenatal female volunteers attending the University of Benin Teaching Hospital, Benin City, Nigeria between June and December 2005, reported an HCV prevalence of $1.86 \%$. Pandolfi Passos et al. (29) performed a cross-sectional study and determined the prevalence and risk factors for HCV infection in 409 patients (248 women, 161 men) attending the infertility clinic at the Hospital de Clinic, Porto Alegre, a teaching hospital in southern Brazil, between 1997 and 1998.The overall prevalence of anti-HCV was 3.2\% among the women and 3.7\% 
among the men. The mean age of the male patients was $33.9 \pm 5.7$ years (range 21-57); the mean age of the female patients was $31.6 \pm 4.1$ years (range 17-39). All subjects were negative for HBV and HIV. They recommend that infertile patients be screened before assisted reproductive techniques.

Pirwany et al. (11) performed a retrospective cohort study of 25 couples undergoing in vitro fertilization and embryo transfer (IVF/ET) cycles in HBV and HCV discordant couples. Thirteen patients were discordant for HBV (10 males and 3 females), and 12 (9 males and 3 females) for HCV. Twenty-seven consecutive age matched patients comprised the control group. The study showed that couples discordant for HBV or HCV had significantly poorer implantation and pregnancy rates $(7.7 \%, 0.0 \%$. respectively) compared with age-matched controls (41\%). Alavian et al. (8) conducted a systematic review to estimate the prevalence of $\mathrm{HBV}$ infection in Iran as accurately as possible for further planning and ways to control the infection. Meta-analysis and survey data analysis of all descriptive/analytical and cross-sectional studies/surveys from April 2001 to March 2007 of the Iranian general population with positive HBsAg were preformed. Fourteen studies met the inclusion criteria. They were from 7 (out of 30) provinces in which approximately $40 \%$ of the Iran's population lives. The HBsAg positive prevalence rates in these provinces were: Golestan (6.3\%), Tehran (2.2\%), East Azarbaijan (1.3\%), Hamadan (2.3\%), Isfahan (1.3\%), Kermanshah (1.3\%) and Hormozgan (2.4\%). The prevalence of HBV infection in Iran is estimated to be $2.14 \% \mathrm{t}$ (95\% CI: 1.92 2.35), for men and $2.55 \%$ for women (95\% CI: 2.25-2.85) and 2.03 percent (95\% CI:1.6-2.46\%), respectively.

Lam et al. (9) examined a retrospective cohort study for the prevalence of HBV infection and the outcomes of the first IVF and embryo transfer treatment cycles in 287 couples undergoing IVF and embryo transfer cycles at the Assisted Reproduction Technology (ART) unit (the tertiary referral center affiliated with the Department of Obstetrics and Gynecology, The Chinese University of Hong Kong ). Results showed that 29 women (10.1\%) were HBV seropositive, whereas 32 (11.1\%) of their husbands were HBV seropositive. Concerning the causes of infertility, there was a trend toward more tubal blockage (57.1\% vs. 42.2\%) in the HBV-infected group. Among the 190 women undergoing their first IVF and embryo transfer cycles, both the ongoing PR or live birth rate and implantation rate in the HBV group were significantly higher than the controls (53.3\% vs. $24.2 \%$ per cycle with embryo transfer; and $43.3 \%$ vs. $18.4 \%$, respectively). They concluded that further studies to elucidate the underlying mechanisms are warranted.

Another study was conducted on 80 pregnant women from January 1999 to June 2000, this investigated the rate of hepatitis $C$ antibodies in pregnant women who had been referred to the Amir Kabir and Razi Ahvaz Hospitals in Iran. Blood samples were taken, after separation of the serum the ELISA test for the detection of the hepa- titis $C$ virus antibody was performed. For those who had serum testing which was positive for hepatitis $\mathrm{C}$, an antibody test was performed confirmatory Immune blat[The immunoblot (Liatek) test was used for confirmation-Organon Liatek Hcv III ]. Five patients (25.6\%) tested positive for the hepatitis $C$ antibody but an ELISA test to confirm was negative. They concluded that the prevalence of the hepatitis $\mathrm{C}$ antibody in pregnant women in Ahwaz City was very low (30).

In assisted reproduction, $\mathrm{HCV}$ transmission may pose a risk for the baby, technicians, and gametes or embryos from non-contaminated parents (31). Alavian et al. (13) selected descriptive and analytic cross-sectional studies and surveys related to the prevalence of HCV infection in the general Iranian population between 2001 and 2008. From the 6,431 studies, eight eligible studies reported a prevalence of HCV infection. They were from six provinces, where approximately $43 \%$ of the country's population lives. They calculated that the HCV infection prevalence rate in Iran was $0.16 \%$ (95\% confidence interval [CI]: 0\%$0.59 \%$ ). Chronic infection with $\mathrm{HCV}$ is a major cause of cirrhosis and hepatocellular carcinoma.

HCV genotyping plays an important role in epidemiological studies of HCV infection. Results of a study to estimate the HCV genotype prevalence in Shiraz revealed that the highest level of infection belonged to 3a followed by 1a in the 634 study participants. Since a considerable proportion of the chronic hepatic $C$ infected patients were intravenous drug abusers in the region in the present findings, it seems advisable to distribute free disposable syringes among the drug injecting addicts both in prison and in their local user areas in the community (32). Some studies evaluating the impact of chronic HCV on hepatic, maternal, and fetal outcomes have suggested a number of potential problems. The infants of HCV-positive mothers were more likely to be of low birth weight, small for gestational age, need assisted ventilation, or require neonatal intensive care.

HCV-positive mothers with excess weight gain had an increased risk of gestational diabetes. Vertical transmission of the hepatitis $C$ virus does occur, but it appears to be much less efficient than for hepatitis B. Early diagnosis of infection in newborns requires HCV-RNA testing. Moreover, our results showed low frequency of hepatitis $\mathrm{B}$ and $\mathrm{C}$ infections in this limited population of infertile couples, but given the low awareness of patients and staff and less support from facilities such as health insurance and a greater likelihood of transferring these infections from patients to other patients and personnel and even from mother to fetus, continuous screening of couples attending IVF clinics is necessary.

Although the prevalence of HIV infection was reported to be zero in this community, screening clients seems appropriate and statistical analysis for HIV in the larger sample size is recommended. It is important to determine HBV, HCV and HIV prevalence among infertile couples who seek assisted reproduction so that specific 
health policies can be devised and infected infertile patients can be counseled during ART cycles. The detection of HBsAg, and anti-HCV must be included as part of routine infertility investigation. In this study, in which couples were evaluated before ART procedures, the prevalence of HCV was in agreement with the local prevalence and data from the literature.

We have concluded that the prevalence of HBsAg, HCV \& HIV antibodies among infertile couples was lower than in the general population and our data is in accordance with other reports. This information may be useful for counseling and management of couples who seek assisted reproduction, and also for further studies analyzing the risk for HBV, HCV and HIV transmission in infertile couples. The possible transmission of these viruses from mother to fetus supports a recommendation for the generalized screening of all infertile couples. Improving mothers' health and well-being enhances not only their lives, but it also contributes to the health of their children and improves community welfare.

\section{Acknowledgments}

The authors would like to thank vice chancellor for research affairs of Ahvaz Jundishapur university of Medical Sciences. This paper is issued from thesis of Farzam Firoozian and financial support was provided by Ahvaz Jundishapur university of Medical Sciences. The research protocol was approved by the Ethics Committee of the Ahvaz Jundishapur University of Medical Sciences.

\section{Financial Disclosure}

All authors declare that they have no conflict of Interest.

\section{Funding/Support}

The study was financially supported by Ahvaz Jundishapur University of Medical Sciences (Grant no. U- 89102), Ahvaz, Iran.

\section{References}

1. Aral SO, Cates W, Jr. The increasing concern with infertility. Why now? JAMA. 1983;250 (17):2327-31.

2. Gray RH. Epidemiology of infertility. Curr Opin Obstet Gynecol. 1990;2 (2):154-8.

3. Templeton A, Fraser C, Thompson B. Infertility epidemiology and referral practice Hum Reprod. 1991;6 (10):1391-4.

4. Speroff L, Fritz MA. Clinical gynecologic endocrinology and infertility. Lippincott Williams \& Wilkins; 2005.

5. Recommendations for use of antiretroviral drugs in pregnant HIV-infected women and interventions to reduce perinatal HIV transmission in the United States, by the Perinatal HIV guidelines Working Group. [Nov. 2, 2007; cited]; Available from: www. avert. org/motherchild. htm.

6. Williams CD, Finnerty JJ, Newberry YG, West RW, Thomas TS, Pinkerton JV. Reproduction in couples who are affected by human immunodeficiency virus: medical, ethical, and legal considerations. Am J Obstet Gynecol. 2003;189 (2):333-41.

7. Ocama P, Opio CK, Lee WM. Hepatitis B virus infection: current status. Am J Med. 2005;118 (12):1413.

8. Alavian SM, Hajarizadeh B, Ahmadzad-Asl M, Kabir A, BagheriLankarani K. Hepatitis B Virus infection in Iran: A systematic review. Hepat Mon. 2008;8 (4):281-94.
9. Lam PM, Suen SH, Lao TT, Cheung LP, Leung TY, Haines C. Hepatitis B infection and outcomes of in vitro fertilization and embryo transfer treatment. Fertil Steril. 2010;93 (2):480-5.

10. Van den Eede B. Investigation and treatment of infertile couples: ESHRE guidelines for good clinical and laboratory practice. European Society of Human Reproduction and Embryology. Hum Reprod.1995;10 (5):1246-71.

11. Pirwany IR, Phillips S, Kelly S, Buckett W, Tan SL. Reproductive performance of couples discordant for hepatitis B and C following IVF treatment. J Assist Reprod Genet. 2004;21 (5):157-61.

12. Weigand $K$, Stremmel W, Encke J. Treatment of hepatitis $C$ virus infection. World J Gastroenterol. 2007;13 (13):1897-905.

13. Alavian SM, Ahmadzad-Asl M, Lankarani KB, Shahbabaie MA, Bahrami Ahmadi A, Kabir A. Hepatitis C infection in the general population of Iran: A systematic review. Hepat Mon. 2009;9 (3):211-23.

14. Mboto CI, Andy IE, Eni OI, Jewell AP. Prevalence, Sociodemographic Characteristics and Risk Factors for Hepatitis C Infection among Pregnant Women in Calabar Municipality, Nigeria. Hepat Mon. 2010;10 (2):116.

15. Onakewhor JU, Okonofua FE. Seroprevalence of Hepatitis C viral antibodies in pregnancy in a tertiary health facility in Nigeria. Niger J Clin Pract. 2009;12 (1):65-73.

16. Bendikson KA, Anderson D, Hornstein MD. Fertility options for HIV patients. Curr Opin Obstet Gynecol. 2002;14 (5):453-7.

17. Manigart Y, Rozenberg S, Barlow P, Gerard M, Bertrand E, Delvigne A. ART outcome in HIV-infected patients. Hum Reprod. 2006;21 (11):2935-40.

18. Afrasiabian S, Hajibagheri K, Yousefinejad V, Rezaiee S, Shahmoradi F. The frequency of Toxoplasma and Cytomegalovirus infections in HIV-positive patients in HIV/AIDS counseling and care center in Kurdistan in 1385. Sci J Kurdistan Univ Med Sci. 2008;13 (2):34-41.

19. Daniel SJ, In thorn G, Marshall M. Harrison Sinternal medicine.17th edition. US: MC Graw-Hill 2008, 261-268. this is a textbook (Fauci, AS $\&$ Lane, HC in Harrison's Principles of Internal Medicine). 17th edition-(McGraw Hill NY, 2008), editor. ; 2008.

20. Hargreave TB, Ghosh C. The impact of HIV on a fertility problems clinic. J Reprod Immunol. 1998;41 (1-2):261-70.

21. Prisant N, Tubiana R, Lefebvre G, Lebray P, Marcelin AG, Thibault $\mathrm{V}$, et al. HIV-1 or hepatitis C chronic infection in serodiscordant infertile couples has no impact on infertility treatment outcome. Fertil Steril. 2010;93 (3):1020-3.

22. Frodsham LC, Smith JR, Gilling-Smith C. Assessment of welfare of the child in HIV positive couples. Hum Reprod. 2004;19 (10):2420-3.

23. Townsend CL, Cortina-Borja M, Peckham CS, de Ruiter A, Lyall $\mathrm{H}$, Tookey PA. Low rates of mother-to-child transmission of HIV following effective pregnancy interventions in the United Kingdom and Ireland, 2000-2006. AIDS. 2008;22 (8):973-81.

24. Qian WP, Tan YQ, Chen Y, Peng Y, Li Z, Lu GX, et al. Rapid quantification of semen hepatitis B virus DNA by real-time polymerase chain reaction. World J Gastroenterol. 2005;11 (34):5385-9.

25. Huang JM, Huang TH, Qiu HY, Fang XW, Zhuang TG, Qiu JW. Studies on the integration of hepatitis B virus DNA sequence in human sperm chromosomes. Asian J Androl. 2002;4 (3):209-12.

26. Neisi N, Makvandi M, Samarbaf-Zadeh AR. A study on genotypes of hepatitis B virus among hemodialysis patients in Khuzestan province. Jundishapur J Microbiol. 2011;4 (2):65-70.

27. Mohammad Jaffari R, Saadati N, Vaziri Esfarjani SH, Soorani Yancheshmeh A. A survey of the frequency of HBsAg status in pregnant women attending health centres in Ahvaz, Payesh Health Monitor. J Iranian Inst Health Sci Res. 2004;3 (3):237-44. .

28. Saadati N, Mohammad Jafari R. A survey of the positive HBsAg frequency in pregnancy women referring to health centres of Ahvaz city. Yakhteh. 2008; ;10 (Suppl 1):78.

29. Passos EP, Silveira TR, Salazar CC, Facin AC, Souza CAB, Guerin YLS, et al. Hepatitis $C$ virus infection and assisted reproduction. Hum Reprod. 2002;17 (8):2085-8.

30. Motlagh ME, Makvandi M, Jalali MT. [Prevalence of anti-HCV among pregnant women]. J Qazvin Univ Med Sci. 2001;8 (1):59-63.

31. Levy R, Tardy J, Bourlet T, Cordonier H, Mion F, Lornage J, et al. Transmission risk of hepatitis $C$ virus in assisted reproductive techniques. Human Reproduction. 2000;15 (4):810.

32. Ziyaeyan M, Alborzi A, Jamalidoust M, Badiee P, Moeini M, Kadivar A. Prevalence of hepatitis $C$ virus genotypes in chronic infected patients, southern Iran. JundishapurJ Microbiol. 2011;4 (3):141-6. 